\title{
UN ESTUDIO DEL E-LEARNING PARA ADULTOS EN EDUCACIÓN UNIVERSITARIA A DISTANCIA: UN ANÁLISIS ESTADÍSTICO SOBRE EL RENDIMIENTO DE ESTUDIANTES DE CONTABILIDAD FINANCIERA EN LA UNED
}

\author{
(A STUDY OF ADULT E-LEARNING IN HIGHER DISTANCE EDUCACION: A \\ STATISTICAL ANALYSIS ABOUT STUDENTS' PERFORMANCE IN FINANCIAL \\ ACCOUNTING IN A SPANISH UNIVERSITY FOR DISTANCE LEARNING (UNED)
}

Teresa Carmen Herrador Alcaide

Montserrat Hernández Solís

Universidad Nacional de Educación a Distancia, UNED (España)

\section{RESUMEN}

Este estudio está encaminado a establecer un modelo para medir el rendimiento del e-learning en educación a distancia de adultos, en el área de economía financiera y contabilidad. Como metodología novedosa se aplica la regresión lineal para el contraste de correlaciones entre las variables del modelo. Se puso en marcha una red de innovación docente para el e-learning, en la que se suministraron diversos materiales en red. La recopilación de datos se ha realizado a través de un sondeo de opinión para evaluar la utilidad de 4 elementos que componen los factores de diseño del modelo e-learning aplicado en la UNED. El contraste de la hipótesis nula mediante análisis por regresión lineal multivariante valida el modelo establecido. Si bien, el análisis logístico-binario y el contraste estadístico del grupo de control ponen de manifiesto que el efecto del e-learning sobre el rendimiento no es tan elevado como cabría esperar.

Palabras clave: e-learning en educación a distancia universitaria, rendimiento del e-learning en estudiantes de contabilidad, percepción estudiantil del e-learning, resultados de la enseñanza online, vídeos interactivos en red.

\begin{abstract}
This paper aims to establish a mathematical model to measure the performance of e-learning in adult distance education in the field of financial economics and accounting. As an innovative methodology, a 'linear regression' approach was applied to contrast correlations between variables in the model. A teacher innovation network was implemented for e-learning using various materials. The compilation of data was carried out by means of an opinion survey to evaluate the usage of the four elements that comprised the design factors of the e-learning
\end{abstract}


model applied at UNED. The contrast of a 'Null Hypothesis', e.g. by means of linear regression analysis validated the established model. However, the 'binary logistical analysis' and the statistical contrast of the group demonstrated that the effect of e-learning on performance was not as high as it had been expected.

Keywords: e-learning in university distance education; performance of e-learning in accounting students; student perception of e-learning; results of online teaching; online interactive videos.

\section{PLANTEAMIENTO DE LA INVESTIGACIÓN}

Las nuevas tecnologías de la información se han extendido a todos los ámbitos de nuestra vida cotidiana, convirtiéndose en instrumentos habituales. El entorno académico no escapa a estos cambios. Las nuevas tecnologías permiten a cada estudiante adaptar el aprendizaje a sus necesidades (Reynar, 2007), más aún en el caso de la enseñanza para adultos, en lo que se denomina Adult learning style (Lenou, Hall y Eighmy, 2011), ya que las diferencias entre las personas aumentan con la edad y estas nuevas herramientas proporcionan diferentes estilos, momentos y lugares de aprendizaje. El gap en el uso de las tecnologías entre diferentes franjas de edad se ha ido acortando (Smith y Caruso, 2010), favoreciendo el uso del e-learning. Entre las bondades que se reconocen al e-learning para adultos se citan, entre otras, las siguientes (Mezirow, 1991; MacKeracher, 2004; Knowles, Holton y Swanson, 2005): capacidad autónoma de trabajo, autoaprendizaje, interacción estudiantes-docente, fomento del trabajo en equipo, etc. Esto ha hecho que las universidades adopten nuevos modelos de enseñanza que permitan integrar las nuevas tecnologías de la información y las comunicaciones, especialmente a partir de la aplicación del EEES (Esteve, 2009; Santamaría y Sánchez-Elvira, 2009), configurándose actualmente como un factor determinante de la calidad de las instituciones de educación (Fernández, 2005).

Estudios previos sobre el e-learning avalan la utilidad que los estudiantes le asignan (Amir, Iqbal y Yasin, 1999); sin embargo, son más los estudios teóricos sobre cómo debe hacerse éste que los estudios relativos a las experiencias y resultados de los estudiantes (Bliuc, Goodyear y Ellis, 2007; Sharpe y Benfield, 2005; Sharpe, Benfield, Roberts y Francis, 2006). En concreto, se sabe que la utilización de las comunidades educativas en red ofrece muchas posibilidades pero también implican una nueva forma de educación (Anderson, 2008; Bates, 2008). El desarrollo de las nuevas tecnologías es más rápido que la evolución en los estilos de aprendizaje, por lo que es necesario investigar sobre las necesidades académicas en este campo (Guri-Rosenblit, 2006). Debemos tener en cuenta que además hay que conjugar las expectativas de los docentes y discentes del entorno e-learning, encontrando un 
espacio común satisfactorio para ambos (O `Neill, Singh y O’Donoghue, 2004). En el caso concreto de los estudiantes adultos, estos necesitan sentirse seguros de sus habilidades (Howell, Williams y Lindsay, 2003; Dorch, 2003).

No se trata, por lo tanto, de debatir si son o no útiles estas herramientas; se trata más bien de realizar una valoración cuantitativa de la influencia real del e-learning en el aprendizaje a distancia de adultos. Por ello, el objetivo de este trabajo es analizar si existe una relación estadística entre las utilidades asignadas a dichas herramientas multimedia (e-learning) y el rendimiento académico obtenido por el estudiante, de forma que se ajuste a una función lineal de utilidad.

Para ello, se ha utilizado una red virtual apoyada en tres tipos de herramientas on-line: mini videos, audio clases y Prueba de Evaluación Continua (PEC). Esta red, de innovación docente, se integró en el marco del VI Proyecto de Innovación Docente desarrollado por la Universidad Nacional de Educación a Distancia (UNED).

La investigación se ha desarrollado dentro de un proyecto de Redes de Innovación Docente, aplicado a una asignatura de nueva implantación de estudios de grado en el ámbito de la economía financiera. La red está integrada por un curso on-line, una plataforma virtual educativa y un sistema de web-conference a través de pizarra digital. Ya se sabe que la principal ventaja de estas redes virtuales es que confieren una infraestructura que integra materiales, herramientas y servicios en una sola unidad que funciona de forma rápida, económica y efectiva (Ong, Lai y Wang, 2004). Se ha utilizado el mini video on line (interactivo) frente al tradicional vídeo televisivo, porque los estudios sobre los resultados de este último no son concluyentes (Kozma, 1986; Sorensen y Baylen, 1999), frente a los obtenidos en relación con los vídeos interactivos en red (online videos).

Una de las ventajas de estos últimos es que permiten centrar la atención sólo en las partes del vídeo que son de interés para el estudiante, favoreciendo una mayor interacción entre discentes y docentes. Otra ventaja, es el apoyo del chat a la interactividad del vídeo. Mostramos un ejemplo de estos vídeos interactivos en la figura 1. 


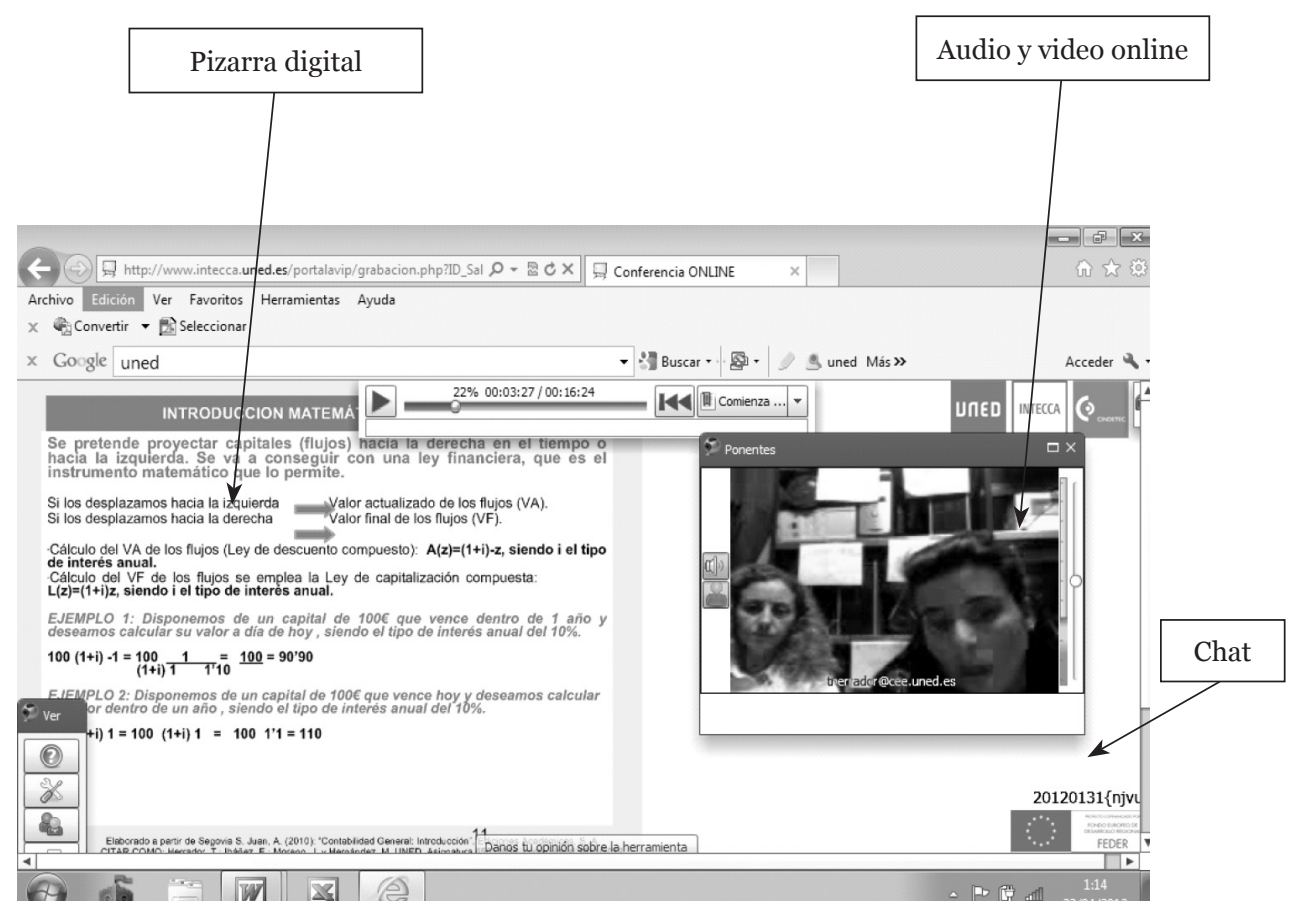

Figura 1. Ejemplo de mini videoactivo online

Fuente: Elaboración propia

También el entorno del e-learning que soporta al vídeo influye en el rendimiento, pudiendo dinamizar el proceso de enseñanza-aprendizaje (Agius y Angelides, 1999). Los docentes que actúan en dicho entorno pueden causar un efecto positivo en el rendimiento académico, principalmente a través de la motivación (Haraway, 2009; Kregor, Breslin y Fountain, 2012). Para hacer posible esta enseñanza online los docentes han desarrollado estrategias creativas individuales para superar los impedimentos, y así, a partir de sus experiencias, han empezado a construir nuevos modelos de enseñanza (Anderson, 2012).

La mayoría de los modelos conceptuales encaminados a investigar los factores que determinan los resultados del e-learning diferencian entre factores humanos (afectan a docentes y discentes) y factores de diseño del sistema (Piccoli, Ahmad e Ives, 2001; Peltier, Drago y Schibrowsky, 2003; Concannon, Flynn y Campbel, 2005; Selim, 2007), estudiando si son determinantes del éxito del e-learning, entre otros, la interacción profesor-alumno, la interacción estudiante-estudiante, la estructura del curso, el contenido del curso, la facilidad de la tecnología usada, la automotivación, la experiencia previa con Internet y los estilos de aprendizaje. Los estudios intentan 
determinar si los factores definidos en el modelo influyen en la percepción del estudiante sobre el entorno e-learning y, en última instancia, en su aprovechamiento y rendimiento académico (Ellis, Ginns y Piggott, 2009).

Para el contraste de modelos se puede utilizar el análisis de la correlación entre los factores que lo integran (Ching, 1998; Eom, Wen y Ashill, 2006), obteniendo los datos del contraste mediante cuestionarios enfocados a la percepción de los discentes en relación al uso de Internet en la educación superior. Este tipo de estudios experimentales ya se han aplicado para evaluar la enseñanza universitaria en economía financiera y contabilidad (Marriott, Marriot y Selwyn, 2004; Hernández, Sánchez y Pra, 2009; Campo y Parte, 2011; Gandía y Montagud, 2011; Herrador y Solís, 2012; López Pérez y otros, 2013 en prensa). Se sabe que la satisfacción atribuida por los estudiantes al e-learning es crucial seguir utilizándolo o abandonarlo (Levy, 2007), y que esta satisfacción está determinada por la utilidad percibida por los estudiantes, además de la calidad en sí del sistema (Roca, Chiu y Martínez, 2006, Roca y Gagne, 2008). Por ello, este estudio, en línea con la literatura específica, se ha centrado en la satisfacción sobre el e-learning, medida por las utilidades asignadas por los estudiantes a cuatro factores/variables que integran el diseño del sistema e-learning de la UNED: el entorno e-learning, los vídeos online (interactivos), las audioclases y la PEC (prueba en línea). Se analiza así si se puede establecer una función de utilidad lineal entre el rendimiento académico y las utilidades asignadas a estos factores. En este estudio no se analiza el efecto de los factores humanos que integran los modelos de e-learning.

Al hilo del planteamiento, resaltamos que justifica más aún la realización de este trabajo, el que éste sea el primero que estudia si se puede establecer una función lineal para cuantificar la utilidad académica del e-learning en el área de economía financiera aplicada a la educación universitaria a distancia para adultos en todo el territorio español, comprobando la utilidad del modelo mediante el contraste de las correlaciones con la técnica de regresión lineal.

\section{METODOLOGÍA DE LA INVESTIGACIÓN}

La red se ha desarrollado en dos fases: una experimental y una analítica.

\section{Metodología de la fase experimental}

En la fase experimental se han desarrollado y distribuido las nuevas herramientas y se ha realizado un sondeo de opinión sobre ellas, siguiendo las fases que mostramos a continuación: 
Selección del colectivo objeto de estudio: Las herramientas aplicadas han sido de libre acceso para los estudiantes de la materia.

Diseño muestral: La población se ha integrado por el conjunto de alumnos presentados a examen, que tuvieron acceso al e-learning a través de diversas herramientas y a quienes les fue distribuido el cuestionario de sondeo de opinión. Dicho cuestionario consta de preguntas breves y cerradas, estructuradas en diversos bloques temáticos. Las respuestas al cuestionario siguen una escala de Likert (1 a 5 puntos).

Los estudiantes que han participado en el sondeo de opinión son 62 alumnos, que representan el 50,4\% sobre el total de presentados a examen. De estos, un $35,42 \%$ son hombres y el resto mujeres.

\section{Metodología de la fase analítica}

En la fase analítica se ha realizado el análisis para establecer si existe relación estadística entre el rendimiento estudiantil y las herramientas online, a través de técnicas de regresión lineal.

La metodología de la fase analítica ha implicado varias etapas concatenadas:

Tratamiento de datos: Se ha realizado la codificación de los datos obtenidos del sondeo de opinión, con un sistema de doble grabación ciega y controles de grabación de datos (controles de rango y grafo, controles cruzados entre varios campos y controles puntuales de cuestionarios).

Análisis estadístico por técnicas de regresión lineal: El objetivo ha sido analizar si se puede establecer una dependencia lineal entre el rendimiento académico del estudiante (medido por la nota obtenida en el examen) y un conjunto de variables independientes: la calificación de cada estudiante en la PEC, el nivel de utilidad que cada uno de ellos ha asignado a las audio-clases, el asignado a los mini videos y el asignado a la utilidad general de la red.

Se ha realizado:

- Un análisis por regresión lineal múltiple,

- Un análisis por regresión bivariante,

- Un análisis logístico-binario, y

- Un contraste estadístico con el grupo de control. 
Para la realización de las técnicas de regresión se emplea el software estadístico específico SPSS, trabajando con un nivel de significación del 5\% (intervalo de confianza del 95\%), que representa el valor para el cual el estadístico de contraste delimita la zona de aceptación o rechazo de la hipótesis nula. Se establece como hipótesis nula, tanto para la regresión lineal múltiple, como para las regresiones lineales bivariantes, la siguiente:

$\mathrm{H}_{\mathrm{o}}$ : La varianza explicada por la regresión seas nula (toda la varianza seas la del error ovarianza residual). Es decir, queno exista dependencia lineal entre las variables objeto de análisis (Independencia lineal).

Si el valor del estadístico de contraste es superior al nivel de significación con el que se va a trabajar en el estudio, estamos en la zona de aceptación de la hipótesis nula, mientras que si el valor del estadístico de contraste es inferior al nivel de significación fijado en el estudio, estamos en la zona de rechazo de la hipótesis nula.

\section{Contraste estadístico con grupo de control}

En este caso se van a tener en consideración las calificaciones obtenidas por los alumnos que no han utilizado ninguno de los materiales multimedia dispuestos en esta red de innovación. Se va a comparar el rendimiento de estos estudiantes con el obtenido por aquellos que sí los emplearon.

\section{ANÁLISIS POR REGRESIÓN LINEAL MÚLTIPLE}

El análisis por regresión lineal múltiple se ha realizado sin desglosar por sexos y posteriormente distinguiendo entre mujeres y hombres. El modelo de regresión lineal múltiple se corresponde con la siguiente expresión matemática:

$$
\mathrm{Y}_{\mathrm{t}}=\mathrm{B}_{0}+\mathrm{B}_{1} \mathrm{X}_{1}+\mathrm{B}_{2} \mathrm{X}_{2}+\mathrm{B}_{3} \mathrm{X}_{3}+\mathrm{B}_{4} \mathrm{X}_{4}+\varepsilon
$$

Donde Yt es la variable explicada (rendimiento obtenido por la nota) en un período de tiempo; $\mathrm{B}_{0}$ es el término constante; $\mathrm{B}_{1}$ el parámetro que mide la influencia del término independiente $\left\langle<\operatorname{PEC}\left(\mathrm{X}_{1}\right)>>, \mathrm{B}_{2}\right.$ el parámetro que mide la influencia del término independiente $<<$ Utilidad asignada a mini-vídeos $\left(\mathrm{X}_{2}\right)>>$; $\mathrm{B}_{3}$ el parámetro que mide la influencia del término independiente $<<$ Utilidad asignada a audio-clases $\left(\mathrm{X}_{3}\right)>>; \mathrm{B}_{4}$ el parámetro que mide la influencia del término independiente $<<$ Utilidad asignada por el estudiante al conjunto de los materiales multimedia $\left(\mathrm{X}_{4}\right)>>$ y por último $\varepsilon$, que es el término aleatorio. En general, si $\mathrm{B}_{\mathrm{i}}>0$, existe correlación lineal positiva entre las variables. 
Los datos obtenidos por el SPSS se muestran en la figura 2:

\begin{tabular}{|c|c|c|c|c|c|}
\hline Modelo & $\mathrm{R}$ & $\mathrm{R}$ cuadrado & $\begin{array}{c}\text { R cuadrado } \\
\text { corregida }\end{array}$ & $\begin{array}{l}\text { Error típ. de } \\
\text { la estimación }\end{array}$ & \\
\hline 1 & $0,460^{a}$ & 0,211 & 0,156 & 1.96428 & \\
\hline \multicolumn{6}{|c|}{ ANOVA $^{b}$} \\
\hline Modelo & $\begin{array}{l}\text { Suma de } \\
\text { cuadrados }\end{array}$ & Gl & $\begin{array}{l}\text { Media } \\
\text { cuadrática }\end{array}$ & $\mathrm{F}$ & Sig. \\
\hline Regresión & 58,922 & 4 & 14,731 & 3,818 & $0,008^{a}$ \\
\hline Residual & 219,928 & 57 & 3,858 & & \\
\hline Total & 278,850 & 61 & & & \\
\hline
\end{tabular}

Figura 2. Resumen del modelo

Fuente: Elaboración propia

El valor del estadístico de contraste es de 0.008 , lo que significa que para un intervalo de confianza del 95\% la regresión lineal multivariante sin desglosar por sexos, se encuentra en la zona de rechazo de la hipótesis nula definida con anterioridad. Es decir, existe dependencia lineal, aunque baja, entre el rendimiento académico y los factores multimedia del entorno e-learning. El siguiente gráfico representa el ajuste a la recta de regresión.

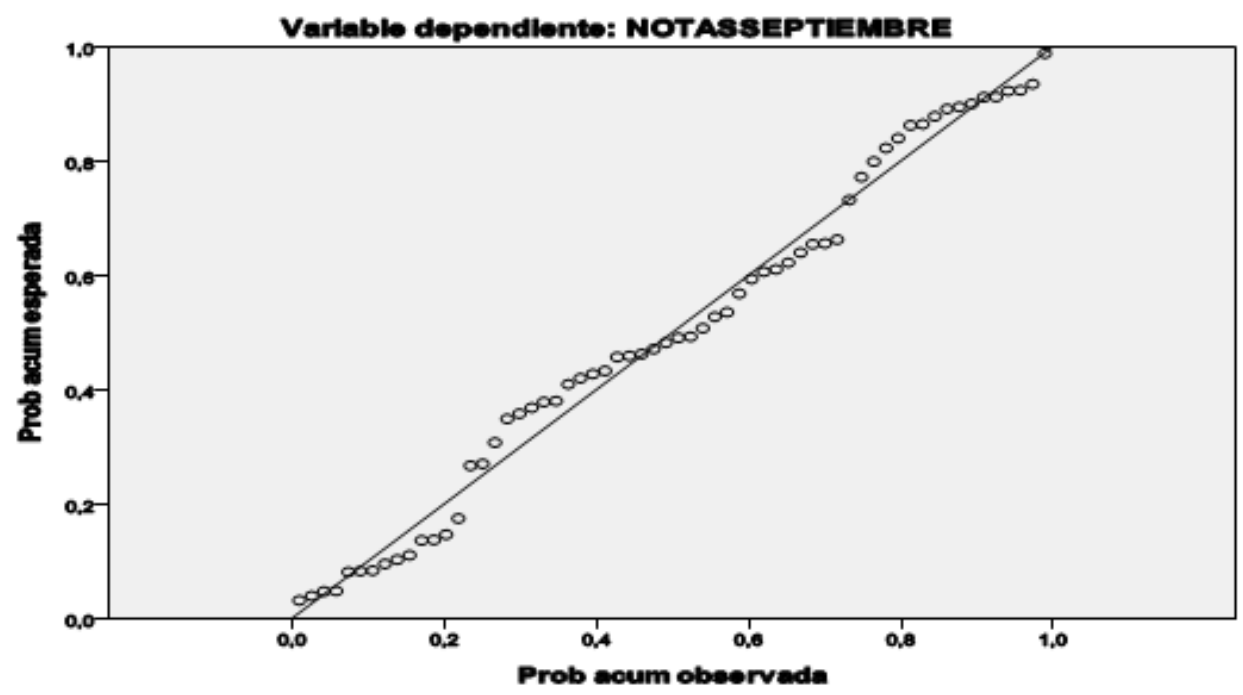

Figura 3. Ajuste lineal del modelo 
A continuación se analiza si la variable "sexo" influye en el rendimiento académico del modelo e-learning de la UNED. Para ello, se estudia la regresión lineal segregando los datos por sexo.

\section{Resultados de la regresión lineal multivariante para el caso de los hombres}

En el caso de los hombres hay un 36\% de concausalidad y un valor del coeficiente de correlación lineal de o.6, lo que implica que la nota del examen se puede explicar a través de una recta de regresión con las cuatro variables predictoras, teniendo una probabilidad elevada de ajuste. Así se puede observar en los valores y el gráfico que se muestra a continuación.

\begin{tabular}{|c|l|l|l|l|}
\hline \multicolumn{1}{|c|}{ Modelo } & \multicolumn{1}{|c|}{$\mathrm{R}$} & \multicolumn{1}{c|}{ R cuadrado } & \multicolumn{1}{c|}{$\begin{array}{c}\text { R cuadrado } \\
\text { corregida }\end{array}$} & $\begin{array}{c}\text { Error típ. de la } \\
\text { estimación }\end{array}$ \\
\hline 1 &, $600^{\mathrm{a}}$ &, 360 &, 209 & 2.26267 \\
\hline
\end{tabular}

Figura 4. Resumen del modelo con segregación para hombres

Fuente: Elaboración propia

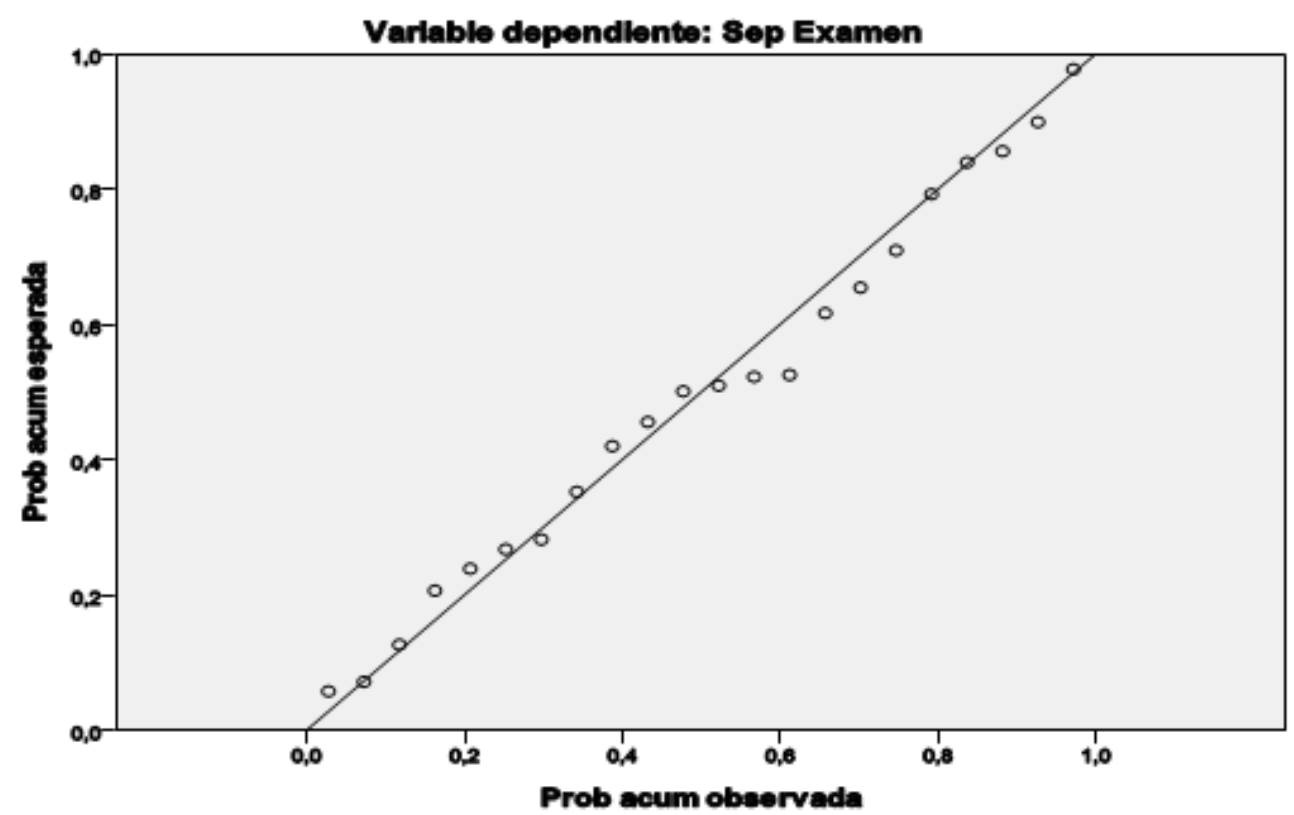

Figura 5. Ajuste lineal del modelo con segregación para hombres 


\section{Resultados de la regresión lineal multivariante para el caso de las mujeres}

Resumen del modelo ${ }^{\mathrm{b}}$

\begin{tabular}{|l|l|l|l|l|}
\hline \multicolumn{1}{|c|}{ Modelo } & \multicolumn{1}{|c|}{$\mathrm{R}$} & \multicolumn{1}{|c|}{ R cuadrado } & \multicolumn{1}{c|}{$\begin{array}{c}\text { R cuadrado } \\
\text { corregida }\end{array}$} & $\begin{array}{c}\text { Error típ. de la } \\
\text { estimación }\end{array}$ \\
\hline 1 & $0,322^{\mathrm{a}}$ & 0,104 & 0,001 & 1.90986 \\
\hline $\begin{array}{l}\text { a. Variables predictoras: (Constante), Utilidad audioclases, PEC, Utilidad General, Utilidad mini } \\
\text { videos } \\
\text { b. Variable dependiente: Sep Examen }\end{array}$
\end{tabular}

Figura 6. Parámetros resumen del modelo con segregación para mujeres Fuente: Elaboración propi

Para el caso de las mujeres, el porcentaje de causas comunes desciende hasta el 10.4\%, mientras que el valor del coeficiente de correlación lineal se sitúa en 0.32, lo que implica un peor ajuste de la recta. En ambos casos la correlación es positiva pero no perfecta, aunque el comportamiento que han tenido los hombres en cuanto a la nota de septiembre se ajusta bastante mejor en forma de recta que para las mujeres, teniendo en cuenta las cuatro variables predictoras con las que se trabaja. Así se observa la conclusión obtenida a través del gráfico de la recta ajustada mediante puntos.

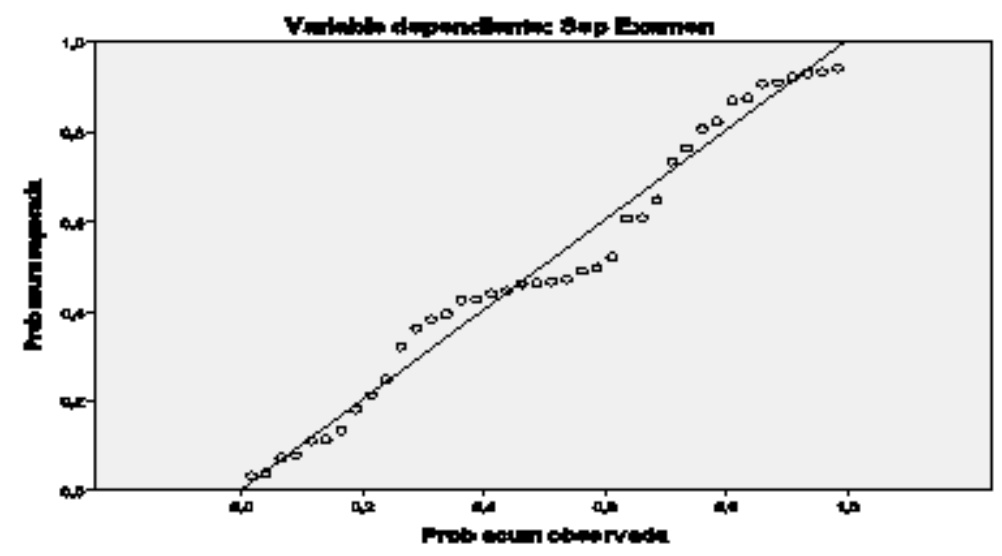

Figura 7. Ajuste del modelo con segregación para mujeres Fuente: Elaboración propia 


\section{ANÁLISIS POR REGRESIÓN LINEAL BIVARIANTE}

El análisis realizado por regresión lineal multivariante se apoya en 4 variables independientes. Profundizamos en la incidencia individual de cada una de estas variables, para ver cuál o cuáles son las que mayor dependencia lineal presentan sobre el rendimiento obtenido por el alumno. Es decir, se realizan regresiones lineales bivariantes, distinguiendo entre mujeres y hombres (segregando por "sexo"), para determinar cuál de las variables independientes es la que más efecto de linealidad ha tenido sobre la variable dependiente. De esta forma, se ha realizado una regresión lineal entre la nota obtenida por el alumno en el examen y:

- La PEC.

- La utilidad que le ha generado el conjunto de los materiales multimedia.

- La utilidad que le ha generado el empleo de los mini videos interactivos.

- La utilidad que le ha generado el empleo de las audio-clases.

Datos obtenidos para la regresión lineal bivariante para el caso de las mujeres:

Nota del examen-PEC

\begin{tabular}{|c|c|c|c|c|c|c|}
\hline Modelo & $\mathrm{R}$ & $\mathrm{R}$ cuadrado & $\begin{array}{l}\mathrm{R} \text { cuadrado } \\
\text { corregida }\end{array}$ & \multicolumn{3}{|c|}{ Error típ. de la estimación } \\
\hline 1 & $0,081^{a}$ & 0,007 & $-0,020$ & \multicolumn{3}{|l|}{1.9295} \\
\hline \multicolumn{7}{|c|}{$\begin{array}{l}\text { a. Variables predictoras: (Constante), Nota.PEC } \\
\text { b. Variable dependiente: Sep Examen }\end{array}$} \\
\hline \multicolumn{7}{|c|}{ ANOVA $^{b}$} \\
\hline \multicolumn{2}{|c|}{ Modelo } & $\begin{array}{c}\text { Suma de } \\
\text { cuadrados }\end{array}$ & $\mathrm{gl}$ & $\begin{array}{c}\text { Media } \\
\text { cuadrática }\end{array}$ & $\mathrm{F}$ & Sig. \\
\hline \multirow{3}{*}{$\frac{\mathrm{H}}{\mathrm{r}}$} & esión & 0,941 & 1 & 0,941 & 0,253 & $0,618^{\mathrm{a}}$ \\
\hline & dual & 141,467 & 38 & 3,723 & & \\
\hline & & 142,408 & 39 & & & \\
\hline
\end{tabular}


Para esta primera regresión lineal bivariante nos encontramos en la zona de aceptación de la hipótesis nula, esto es, que existe independencia lineal entre las variables. Así lo confirma el valor de R, con sólo un $7 \%$ de causas comunes.

\section{Nota del examen-Utilidad Global}

\begin{tabular}{|l|l|l|l|l|}
\hline Modelo & \multicolumn{1}{|c|}{$\mathrm{R}$} & \multicolumn{1}{|c|}{ R cuadrado } & $\begin{array}{c}\text { R cuadrado } \\
\text { corregida }\end{array}$ & \multicolumn{1}{|c|}{ Error típ. de la estimación } \\
\hline 1 & o,303 $^{\mathrm{a}}$ & 0,092 & 0,068 & 1.8446 \\
\hline
\end{tabular}

a. Variables predictoras: (Constante), Utilidad General

b. Variable dependiente: Sep Examen

\begin{tabular}{|l|l|l|l|l|l|c|}
\hline \multicolumn{7}{|c|}{ ANOVA $^{\mathrm{b}}$} \\
\hline 1 & $\begin{array}{c}\text { Suma de } \\
\text { cuadrados }\end{array}$ & \multicolumn{1}{|c|}{ gl } & $\begin{array}{c}\text { Media } \\
\text { cuadrática }\end{array}$ & F & Sig. \\
\hline & Regresión & 13,104 & 1 & 13,104 & 3,851 & $0^{2}, 057^{\mathrm{a}}$ \\
\hline & Residual & 129,304 & 38 & 3,403 & & \\
\hline & Total & 142,408 & 39 & & & \\
\hline $\begin{array}{l}\text { a. Variables predictoras: (Constante), Utilidad General } \\
\text { b. Variable dependiente: Sep Examen }\end{array}$
\end{tabular}

Figura 9. Resumen del modelo bivariante $<<$ Utilidad global-mujeres $>>$

Fuente: Elaboración propia

Se acepta la hipótesis nula, es decir, la independencia lineal entre las variables. El valor del coeficiente de determinación lineal es muy bajo, con sólo un $9 \%$ de concausalidad entre las dos variables.

Nota de examen-Utilidad de los Mini videos

\begin{tabular}{|c|c|c|c|c|c|c|}
\hline Modelo & $\mathrm{R}$ & R cuadrado & $\begin{array}{l}\text { R cuadrado } \\
\text { corregida }\end{array}$ & \multicolumn{3}{|c|}{ Error típ. de la estimación } \\
\hline 1 & $0,220^{a}$ & 0,048 & 0,023 & \multicolumn{3}{|l|}{1.8886} \\
\hline \multicolumn{7}{|c|}{$\begin{array}{l}\text { a. Variables predictoras: (Constante), Utilidad mini videos } \\
\text { b. Variable dependiente: Sep Examen }\end{array}$} \\
\hline \multicolumn{7}{|c|}{ ANOVA $^{\text {b }}$} \\
\hline \multicolumn{2}{|c|}{ Modelo } & $\begin{array}{l}\text { Suma de } \\
\text { cuadrados }\end{array}$ & $\mathrm{gl}$ & $\begin{array}{l}\text { Media } \\
\text { cuadrática }\end{array}$ & $\mathrm{F}$ & Sig. \\
\hline \multirow[t]{3}{*}{1} & gresión & 6,875 & 1 & 6,875 & 1,927 &, $173^{\mathrm{a}}$ \\
\hline & sidual & 135,533 & 38 & 3,567 & & \\
\hline & tal & 142,408 & 39 & & & \\
\hline
\end{tabular}




\begin{tabular}{|c|c|c|c|c|c|c|}
\hline Modelo & $\mathrm{R}$ & R cuadrado & $\begin{array}{l}\text { R cuadrado } \\
\text { corregida }\end{array}$ & \multicolumn{3}{|c|}{ Error típ. de la estimación } \\
\hline 1 & $0,220^{a}$ & 0,048 & 0,023 & \multicolumn{3}{|l|}{1.8886} \\
\hline \multicolumn{7}{|c|}{$\begin{array}{l}\text { a. Variables predictoras: (Constante), Utilidad mini videos } \\
\text { b. Variable dependiente: Sep Examen }\end{array}$} \\
\hline \multicolumn{7}{|c|}{ ANOVA $^{b}$} \\
\hline \multicolumn{2}{|c|}{ Modelo } & $\begin{array}{l}\text { Suma de } \\
\text { cuadrados }\end{array}$ & gl & $\begin{array}{c}\text { Media } \\
\text { cuadrática }\end{array}$ & $\mathrm{F}$ & Sig. \\
\hline \multirow[t]{2}{*}{1} & gresión & 6,875 & 1 & 6,875 & 1,927 &, $173^{\mathrm{a}}$ \\
\hline & sidual & 135,533 & 38 & 3,567 & & \\
\hline \multicolumn{7}{|c|}{$\begin{array}{l}\text { a. Variables predictoras: (Constante), Utilidad mini vídeos } \\
\text { b. Variable dependiente: Sep Examen }\end{array}$} \\
\hline
\end{tabular}

Figura 10. Resumen del modelo bivariante $<<$ Utilidad mini vídeos-mujeres $>>$ Fuente: Elaboración propia

De nuevo se acepta la hipótesis de independencia lineal entre las variables y un porcentaje de causas comunes de linealidad que bajan al $4 \%$.

Nota de examen- utilidad audio-clases

\begin{tabular}{|c|c|c|c|c|c|c|c|}
\hline \multicolumn{2}{|c|}{ Modelo } & $\mathrm{R}$ & R cuadrado & $\begin{array}{c}\text { R cuadrado } \\
\text { corregida }\end{array}$ & \multicolumn{3}{|c|}{ Error típ. de la estimación } \\
\hline \multicolumn{2}{|c|}{1} & $0,229^{a}$ & 0,052 & 0,027 & \multicolumn{3}{|l|}{1.8845} \\
\hline \multicolumn{8}{|c|}{$\begin{array}{l}\text { a. Variables predictoras: (Constante), Utilidad audioclases } \\
\text { b. Variable dependiente: Sep Examen }\end{array}$} \\
\hline \multicolumn{8}{|c|}{ ANOVA $^{b}$} \\
\hline \multicolumn{3}{|c|}{ Modelo } & $\begin{array}{l}\text { Suma de } \\
\text { cuadrados }\end{array}$ & $\mathrm{gl}$ & $\begin{array}{c}\text { Media } \\
\text { cuadrática }\end{array}$ & $\mathrm{F}$ & Sig. \\
\hline \multirow[t]{3}{*}{1} & \multicolumn{2}{|c|}{ Regresión } & 7,461 & 1 & 7,461 & 2,101 &, $155^{\mathrm{a}}$ \\
\hline & \multicolumn{2}{|c|}{ Residual } & 134,946 & 38 & 3,551 & & \\
\hline & \multicolumn{2}{|c|}{ Total } & 142,408 & 39 & & & \\
\hline
\end{tabular}

Figura 11. Resumen del modelo bivariante $<<$ Utilidad Audioclases-mujeres $>>$ Fuente: Elaboración propia

Se vuelve a estar en la zona de aceptación de la hipótesis nula, dado que Sig es superior al 0.05. Se admite la independencia lineal entre las variables, dado que un porcentaje de concausalidad del $5 \%$ es bajo. 


\section{Datos obtenidos para la regresión lineal bivariante para el caso de los hombres}

Nota de examen-PEC

\begin{tabular}{|c|c|c|c|c|c|c|}
\hline Modelo & $\mathrm{R}$ & R cuadrado & $\begin{array}{c}\mathrm{R} \text { cuadrado } \\
\text { corregida }\end{array}$ & \multicolumn{3}{|c|}{ Error típ. de la estimación } \\
\hline 1 & $0,022^{a}$ & 0,001 & $-0,049$ & \multicolumn{3}{|l|}{2.6066} \\
\hline \multicolumn{7}{|c|}{ ANOVA $^{b}$} \\
\hline \multicolumn{2}{|c|}{ Modelo } & $\begin{array}{l}\text { Suma de } \\
\text { cuadrados }\end{array}$ & $\mathrm{gl}$ & $\begin{array}{c}\text { Media } \\
\text { cuadrática }\end{array}$ & $\mathrm{F}$ & Sig. \\
\hline \multirow{3}{*}{$\frac{\mathrm{r}}{\mathrm{r}}$} & resión & , 069 & 1 & 0,069 & 0,010 & $0,921^{a}$ \\
\hline & dual & 135,884 & 20 & 6,794 & & \\
\hline & & 135,953 & 21 & & & \\
\hline
\end{tabular}

Figura 12. Resumen del modelo bivariante $<<$ Nota PEC-hombres $>>$

Fuente: Elaboración propia

En este caso el nivel de dependencia lineal bivariante es escasísimo, sólo un 1\%, por lo que no se puede explicar la nota obtenida en el examen de septiembre en base a la nota obtenida en la PEC.

Nota de examen-Utilidad Global

\begin{tabular}{|c|c|c|c|c|c|c|}
\hline \multicolumn{2}{|c|}{ Modelo } & $\begin{array}{l}\text { Suma de } \\
\text { cuadrados }\end{array}$ & gl & $\begin{array}{c}\text { Media } \\
\text { cuadrática }\end{array}$ & $\mathrm{F}$ & Sig \\
\hline \multicolumn{7}{|c|}{ ANOVA $^{b}$} \\
\hline \multirow[t]{3}{*}{1} & Regresión & 41,942 & 1 & \begin{tabular}{|l|}
41,942 \\
\end{tabular} & 8,923 & $0,007^{\mathrm{a}}$ \\
\hline & Residual & 94,011 & 20 & 4,701 & & \\
\hline & Total & 135,953 & 21 & & & \\
\hline
\end{tabular}

Figura 13. Anova del modelo bivariante <<Utilidad global-hombres $>>$ Fuente: Elaboración propia

Los valores se encuentran en la zona de rechazo de la hipótesis nula, por lo que se admite una dependencia lineal entre la nota del examen y la utilidad general de los materiales multimedia, con un 30\% de causas comunes. Se puede explicar 
el rendimiento obtenido por el alumno según la utilidad global que los materiales multimedia le han proporcionado.

Nota de examen-Utilidad de los mini videos.

\begin{tabular}{|l|l|l|l|l|}
\hline Modelo & \multicolumn{1}{|c|}{$\mathrm{R}$} & \multicolumn{1}{|c|}{ R cuadrado } & $\begin{array}{c}\text { R cuadrado } \\
\text { corregida }\end{array}$ & \multicolumn{1}{|c|}{ Error típ. de la estimación } \\
\hline 1 & $0,504^{\mathrm{a}}$ & 0,254 & 0,217 & 2.2520 \\
\hline
\end{tabular}

a. Variables predictoras: (Constante), Utilidad mini vídeos

b. Variable dependiente: Examen

\begin{tabular}{|c|c|c|c|c|c|c|}
\hline \multicolumn{7}{|c|}{ ANOVA $^{b}$} \\
\hline \multicolumn{2}{|c|}{ Modelo } & Suma de & gl & Media & $\mathrm{F}$ & Sig. \\
\hline 1 & Regresión & 34,520 & 1 & 34,520 & 6,807 & $0,017^{\mathrm{a}}$ \\
\hline & Residual & 101,432 & 20 & 5,072 & & \\
\hline & Total & 135,953 & 21 & & & \\
\hline
\end{tabular}

Figura 14. Resumen del modelo bivariante $<<$ Utilidad mini vídeos-hombres $>>$ Fuente: Elaboración propia

Volvemos a estar en la zona de rechazo de la hipótesis nula. Existe dependencia lineal entre la nota obtenida en el examen de septiembre y la utilidad de los mini videos como material multimedia, con un $25 \%$ de concausalidad.

Nota de examen-Utilidad de las Audio-clases.

\begin{tabular}{|l|l|l|l|l|}
\hline Modelo & \multicolumn{1}{|c|}{$\mathrm{R}$} & \multicolumn{1}{|c|}{ R cuadrado } & $\begin{array}{c}\text { R cuadrado } \\
\text { corregida }\end{array}$ & \multicolumn{1}{|c|}{ Error típ. de la estimación } \\
\hline 1 & $0,554^{\mathrm{a}}$ & 0,307 & 0,272 & 2.17075 \\
\hline
\end{tabular}

a. Variables predictoras: (Constante), Utilidad audioclases

b. Variable dependiente: Ex

\begin{tabular}{|l|l|l|l|l|l|c|}
\hline \multicolumn{7}{|c|}{ ANOVA $^{\mathrm{b}}$} \\
\hline \multicolumn{2}{|c|}{ Modelo } & $\begin{array}{c}\text { Suma de } \\
\text { cuadrados }\end{array}$ & gl & $\begin{array}{c}\text { Media } \\
\text { cuadrática }\end{array}$ & F & Sig. \\
\hline 1 & Regresión & 41,709 & 1 & 41,709 & 8,851 & $0,007^{\mathrm{a}}$ \\
\hline
\end{tabular}




\begin{tabular}{|l|l|l|l|l|l|l|}
\hline & Residual & 94,243 & 20 & 4,712 & & \\
\hline & Total & 135,953 & 21 & & & \\
\hline \\
$\begin{array}{l}\text { a. Variables predictoras: (Constante), Utilidad audioclases } \\
\text { b. Variable dependiente: Sep Examen }\end{array}$
\end{tabular}

Figura 15. Resumen del modelo bivariante $<<$ Utilidad audioclases-hombres $>>$ Fuente: Elaboración propia

Con este segundo material multimedia, se vuelve a estar en la zona de rechazo de la hipótesis nula. Existe dependencia lineal entre la nota obtenida en el examen de septiembre y la utilidad de las audio-clases, con un 30\% de concausalidad.

\section{Conclusión final de las regresiones bivariantes segregadas por sexos}

Son los hombres los que han establecido la dependencia lineal entre la nota obtenida en el examen de septiembre y el empleo del uso de los materiales multimedia: en un 30\% se explica el rendimiento del alumno según la utilidad proporcionada por el conjunto de los materiales multimedia, en un $25 \%$ se explica el rendimiento del alumno segúnla utilidad proporcionada por los mini videos y en un $30.7 \%$ se explica el rendimiento del alumno según la utilidad proporcionada por las audioclases. En cambio, para las mujeres, el porcentaje de concausalidad de la variable dependiente con las variables independientes es prácticamente inexistente, situándose, para cada una de ellas en torno al $5 \%$ de media. Este resultado coincide con el del estudio de Wang, Wu y Wang (2009). Estos autores destacan que la diferencia de género es un factor que puede moderar el uso del m-learning (e-learning por móvil), lo que afecta a las expectativas de éxito del estudiante y esto se traduce en un menor rendimiento.

Para cualquiera de los dos sexos, el rendimiento académico del alumno apenas se puede explicar por la calificación obtenida en la PEC. Por esta razón concluimos que esta prueba no ha influido prácticamente nada a la hora de mejorar su rendimiento en la convocatoria de septiembre. 


\begin{tabular}{|c|c|c|}
\hline $\begin{array}{c}\text { \% CONCAUSALIDAD LINEAL ENTRE EL } \\
\text { RENDIMIENTO DEL ALUMNO Y CADA UNA DE } \\
\text { LAS VARIABLES INDEPENDIENTES: }\end{array}$ & HOMBRES & MUJERES \\
\hline NOTA DE LA PEC & $1 \%$ & $7 \%$ \\
\hline $\begin{array}{c}\text { UTILIDAD GLOBAL DE LOS MATERIALES } \\
\text { MULTIMEDIA }\end{array}$ & $30 \%$ & $9 \%$ \\
\hline UTILIDAD MINI-VÍDEOS & $25 \%$ & $4 \%$ \\
\hline UTILIDAD AUDIO-CLASES & $30.7 \%$ & $5 \%$ \\
\hline
\end{tabular}

Figura 16. Resumen de concausalidad rendimiento estudiantil/variables Fuente: Elaboración propia

Como complemento al análisis por regresión lineal se aplica el análisis logísticobinario, que mostramos a continuación.

\section{ANÁLISIS LOGÍSTICO-BINARIO}

Esta técnica lleva los valores de la variable dependiente o explicada (rendimiento académico $)$ a valores dicotómicos $(\mathrm{O}=$ suspenso y $1=$ aprobado), para establecer sólo si existe o no relación lineal entre ésta y las variables predictoras (independientes), no la proporción de linealidad. Esta técnica de regresión se considera complementaria de las anteriores, dado que se pierde información al trabajar con valores dicotómicos, pero a cambio se puede determinar mejor la existencia de linealidad. A continuación mostramos los resultados obtenidos para hombres y para mujeres.

\begin{tabular}{|l|l|c|c|c|c|c|c|c|}
\hline \multicolumn{7}{|c|}{ Regresión logística binaria mujeres-Variables en la ecuación } \\
\hline \multicolumn{2}{|l|}{} & B & E.T. & Wald & gl & Sig. & Exp(B) \\
\hline Paso o & Constante & 0,405 & 0,323 & 1,578 & 1 & 0,209 & 1,500 \\
\hline Regresión logístico binaria hombres-Variables en la ecuación \\
\hline \multicolumn{2}{|c|}{} & B & E.T. & Wald & gl & Sig. & Exp(B) \\
\hline Paso o & Constante & 0,368 & o,434 & 0,719 & 1 & 0,396 & 1,444 \\
\hline
\end{tabular}

Figura 17. Resumen logístico-binario para hombres

Fuente: Elaboración propia

El análisis bivariante confirma la aceptación de la hipótesis nula para las mujeres, esto es, se acepta la independencia lineal entre la nota obtenida por las mujeres y cada una de las variables independientes. Para los hombres ocurre lo mismo, aceptándose la independencia lineal entre la nota obtenida por el alumno en la convocatoria de septiembre y cada una de las variables independientes por sí mismas. Vemos así como el bajo porcentaje de concausalidad obtenido para los hombres se diluye al 
ser llevado el análisis a valores dicotómicos, lo cual nos permite concluir que las variables "utilidad global de la red", "utilidad de los mini vídeos", "utilidad de las audioclases" y "nota de la PEC", consideradas individualmente, no tienen incidencia en el rendimiento académico. Sin embargo, sí influyen en el rendimiento cuando se consideran de forma conjunta (análisis por regresión lineal múltiple), si bien a niveles bajos. Para contrastar esta baja linealidad multivariante, se ha realizado un contraste con el grupo de control.

\section{CONTRASTE ESTADÍSTICO CON EL GRUPO DE CONTROL}

El grupo de control está compuesto por el alumnado que no ha hecho uso de los recursos didácticos multimedia que se han ofertado en esta red de innovación docente. Los resultados descriptivos obtenidos se muestran a continuación y se comparan con los resultados conseguidos por la muestra de alumnos que si han empleado dichos recursos didácticos.

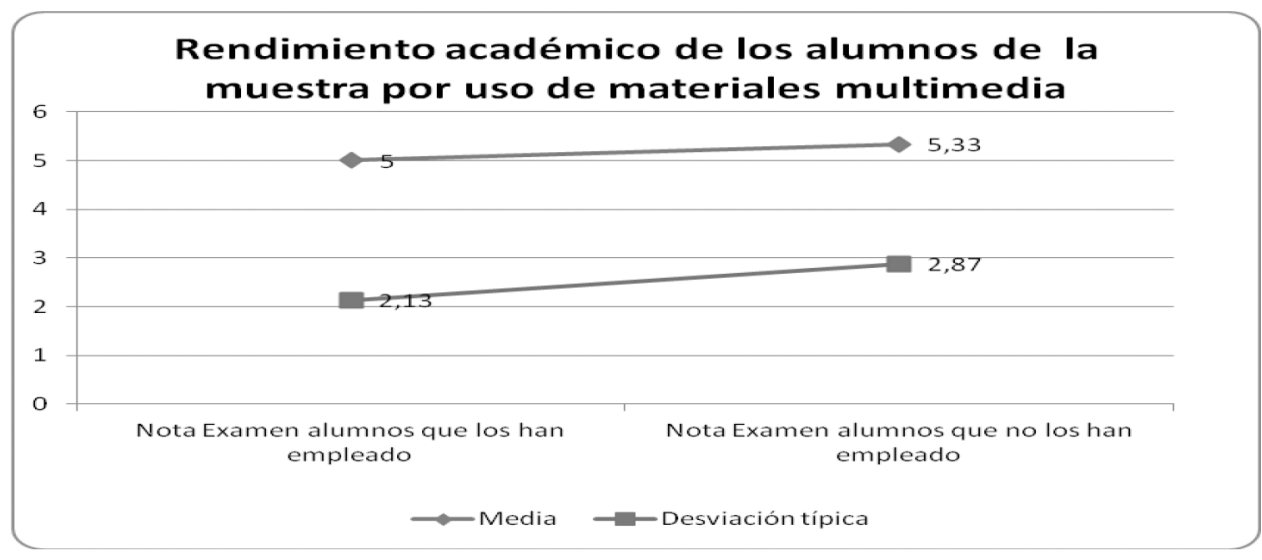




\begin{tabular}{|c|c|c|}
\hline $\begin{array}{c}\text { RESULTADOS DESCRIPTIVOS DEL } \\
\text { RENDIMIENTO ACADÉMICO DE LOS ALUMNOS } \\
\text { QUE HAN EMPLEADOS LOS RECURSOS } \\
\text { MULTIMEDIA }\end{array}$ & MEDIA & $\begin{array}{c}\text { DESVIACION } \\
\text { TIPICA }\end{array}$ \\
\hline EXAMEN DE LA CONVOCATORIA & 5,00 & 2,13 \\
\hline NOTA DE LA PEC & 0,24 & 0,30 \\
\hline UTILIDAD GLOBAL DE LA RED & 3,77 & 1,03 \\
\hline UTILIDAD MINI VIDEOS & 3,82 & 1,10 \\
\hline UTILIDAD AUDIOCLASES & 3,65 & 1,13 \\
\hline $\begin{array}{c}\text { RESULTADOS DESCRIPTIVOS DEL } \\
\text { RENDIMIENTO ACADÉMICO DE LOS ALUMNOS } \\
\text { QUE NO HAN EMPLEADOS LOS RECURSOS } \\
\text { MULTIMEDIA }\end{array}$ & $\mathbf{M E D I A}$ & $\begin{array}{c}\text { DESVIACION } \\
\text { TIPICA }\end{array}$ \\
\hline EXAMEN DE LA CONVOCATORIA & 5,33 & 2,87 \\
\hline
\end{tabular}

Figura 18. Resumen contraste con el grupo de control Fuente: Elaboración propia

A la vista de los resultados, lo primero que cabe decir es que el rendimiento medio de los alumnos se sitúa en torno al aprobado, siendo algo ligeramente superior en el caso de los alumnos que no han hecho uso de los recursos multimedia. Esto indica que el hecho de que el alumno haya estudiado manejando los nuevos materiales multimedia apenas le ha influido en su rendimiento. Este resultado nos lleva a cuestionarnos el porqué. Pensamos que bien porque no le haya dedicado suficiente tiempo, atención y motivación o bien porque haya hecho un uso incorrecto de los mismos (sólo un 15,06\% de los alumnos presentados a examen no han estudiado a través de los recursos didácticos ofertados, frente al 84,93\% restante que si lo han hecho). En este sentido, algunos estudios encontraron que la percepción de utilidad del e-learning se puede ver influenciada por otros ítems, tales como las características propias de estudiantes (tendencia a la participación) o por otros factores como la instrucción multimedia previa (Liaw, 2008).

En la muestra de los alumnos que han estudiado con los materiales multimedia existen más desviaciones de los valores de cada una de las variables independientes con respecto a la media. Se puede observar que la desviación típica, tanto de la utilidad de los mini videos como de las audio clases representan valores de la variable bastante alejados del valor medio y, también sucede con la utilidad global del proyecto de red. 


\section{CONCLUSIONES}

Como principal conclusión de este estudio se soporta estadísticamente que, el efecto conjunto de las 4 variables de diseño del sistema del modelo de e-learning de la UNED tienen cierta incidencia en el rendimiento académico del estudiante. De forma que se puede establecer una función de utilidad lineal entre dicho rendimiento y las variables analizadas. Sin embargo, la consideración del efecto individual de cada una de éstas en el rendimiento es escasa. Esto puede ser porque existen otras variables no consideradas en el modelo que también determinan el rendimiento del estudiante, como podría ser el esfuerzo individual, la formación multimedia, la interacción o la autoestima, encuadradas en las variables humanas de modelos e-learning, que son difíciles de cuantificar.

Un análisis más profundo, mediante la regresión lineal bivariante de cada variable independiente con la dependiente, observamos que la PEC apenas ha incidido en el rendimiento de los estudiantes, tanto para hombres como para mujeres, lo que implica que esta herramienta online apenas les ha sido útil. Sin embargo, el resto de las variables que representan a las demás herramientas online (mini videos, audio clases y la red) han aportado algo de utilidad al rendimiento estudiantil, siendo mucho mayor en el caso de los hombres (véase cuadro correspondiente), por lo que un factor a considerar como variable para futuros estudios es la segregación por sexos. En este sentido se encontraron resultados similares para el caso de los vídeos interactivos (Zhang, Zhou, Robert, Briggs y Nunamaker, 2006).

Para explicar porqué las herramientas e-learning no han tenido un efecto tan elevado como a priori se les atribuye, varios autores manejan diversas posibilidades. La mayoría de ellos lo enfocan a la importancia de la interacción en el Open Distance Learning (ODL) (Anderson, 2003; Boyle y Wambach, 2001; Dzakiria, 2008). Se defiende que a mayor nivel de interactividad se suponen mayores niveles de rendimiento (Michailidou y Economides, 2003; Cheng et al., 2012; Sun, Chen, Lin y Wang, 2008; Robert, Ginns y Piggot, 2009), si bien existen estudios que contrastan que la mayor interacción no garantiza por sí misma un mayor rendimiento (Sun y Hsu, 2012; Davis y Graff, 2005), debiendo diferenciarse entre interacción e interactividad (Wagner, 1994, 1997).

Pensamos que otra variable que podría estar minorando el rendimiento del e-learning es que la cultura multimedia aplicada en la docencia no tiene aún un fuerte arraigo en España, por lo que las herramientas se están infrautilizando. Es conveniente destacar que la edad media de nuestra población, dadas las características de universidad a distancia en la que se encuadra la red, se sitúa en torno a los 40 
años, lo que nos lleva a pensar que no priorizan el entorno multimedia sobre los métodos discentes tradicionales (clases magistrales y libros), y que ya tienen unas expectativas respecto al modelo establecido, como ya contrastaron Chiu y Wang (2008) y Robert, Ginns y Piggot (2009).

Así, con esta metodología basada en la regresión lineal, hemos determinado que se pueden modelizar algunas de las variables que de forma conjunta explican el rendimiento académico del e-learning. Si bien, el modelo necesita ser ajustado, por lo que esperamos encontrar resultados futuros que nos permitan acotar más las variables del denominado rendimiento e-learning, de cara a poder mejorar el modelo e-learning que utilizamos.

\section{REFERENCIAS BIBLIOGRÁFICAS}

Agius, H. W.; Angelides, M. C. (1999). Developing knowledge-based intelligent multimedia tutoring systems using semantic content based modeling. Artificial Intelligence Review, 13 (1), (55-83).

Amir, F.; Iqbal, S. M.; Yassin, M. (1999). Effectiveness of cyberlearnins. Procceeding of the 29th Asee/IEEE frontiers in education conference, San Juan, Puerto Rico, (13a2-7-13a2-12).

Anderson, T. (2003). Getting the mix right again: An updated and theoretical rationale for interaction. International Review of Research in Open and Distance Learning, 4 (2), (1-14).

Anderson, T. (2008). Social software technologies in distance education. Maximizing learning freedom. En Evans, T.; Haughey, M.; Murphy, D. (Eds.). International handbook of distance education. West Anglia U.K.: Emerald Group Publishings, (167-184).

Anderson, T. (2012). Learning technology through three generation of technology enhanced distance education pedagogy. European Journal of Open Distance E-learning. [en línea] Disponible en: http://www.eurodl.org/materials/contrib/2012/Anderson Dron.pdf, [consulta 2012, 13 de septiembre]

Bates, T. (2008). Transforming distance education through new technologies.

En Evans, T.; Haughey, M.; Murphy, D. (Eds.). International handbook of distance education, (217-235). West Anglia U.K.: Emerald Group Publishings.

Bliuc, A.; Goodyear, P.; Ellis, R. A. (2007). Research focus and methodological choices in studies into students' experiences of blended learning in higher education. Internet and Higher Education, 15, (231-244).

Boyle, D.K.; Wambach, K. A. (2001). Interaction in graduate nursing Web-based Instruction. Journal of Professional Nursing, 17, (128-134).

Campo Moreno, P.; Parte Esteban, L. (2011). La Webquest como estrategia metodológica en la enseñanza universitaria de la asignatura de Contabilidad de Empresas Turísticas. EDUTEC. Revista electrónica de tecnología educativa”, 38 [en línea] Disponible en http://edutec.rediris.es/ Revelec2/Revelec38/pdf/Edutec-e 38 DelCampo Parte.pdf, [consulta 2012, 19 de abril].

Cheng, B.; Wang, M.; Moormann, J.; Olarian, B.A.; Chen, N-S. (2012). The Effects of Organizational Learning Environment Factors on E-Learning Acceptance. Computers \& Education, 58 (3), Apr 2012, (885-899).

Chin, W. W. (1998). The partial least squares approach to structural equation 
modeling. En Marcoulides, G. A. (ed.). Modern methods for business research. Mahwah, NJ: Erlbaum, (295-336).

Chiu, C-M.; Wang, E. T. G. (2008). Understanding Web-based learning continuance intention: The role of subjective task value. Information \& Management, 45 (3), (194-201).

Concannon, F.; Flynn, A.; Campbel, M. (2005). What campus-based students think about the quality and benefits of elearning. British Journal of Educational Technology, 36 (3), (501-512).

Davis, J.; Graff, M. (2005). Performance in e-learning: online participation and student grades. British Journal of Educational Technology, 36 (4), (570-663).

Dortch, K. D. (2003). How to get learners to learn, Distance Education and Training Council. Report on the DETC 77th Annual Conference. (13-15).

Dzakiria, H. (2008). Students' Accounts of the Need for Continuous Support in a Distance Learning Programme. Open Learning: The Journal of Open and Distance Learning, 23 (2). Rutledge, Taylor \& Francis Group, (103-112).

Ellis, R. A.; Ginns, P.; Piggott, L. (2009). Elearning in higher education: some key aspects and their relationship to approaches to study". Higher Education Research \& Development, 28 (3), (303318).

Eom, B. S.; Wen, H. J.; Ashill, N. (2006). The Determinants of Students' Perceived Learning Outcomes and Satisfaction in University Online Education: An Empirical Investigation, Decision Sciences Journal of Innovative Education, 4 (2), (215-235).

Esteve, F. (2009). Bolonia y las TIC. De la docencia 1.0 al aprendizaje 2.0. La cuestión universitaria, 5, (59-68). [en línea] Disponible en http://www.lacuestionuniversitaria.upm.es/web/grafica/articulos/imgs boletin 5/pdfs/LCU5-6.pdf). [consulta 2012, 25 de marzo].
Fernández Díaz, M. J. (2005). La innovación como factor de calidad en las organizaciones educativas, Educación XXI, 8, (67-86). [en línea] Disponible en http:// www.uned.es/educacionXX1/pdfs/o803.pdf. [consulta 2012, 23 de abril].

Gandía, J. L.; Montagud, J. L. (2011). Innovación docente y resultados del aprendizaje: Un estudio empírico en la enseñanza de la contabilidad de costes. Revista Española de Financiación y Contabilidad, XI (152), (677-698).

Guri-Rosenblit, S. (2006). Eight paradoxes in the implementation process of e-learning in higher education. Distance et Savior. [en línea] Disponible en http://www.cairn.info/resume.php?ID ARTICLE=DIS 042 0155. [consulta 2012, 11 de abril].

Haraway, D. (2009). A cyborg manifesto. The Cultural Studies Reader. 40. ed. London: During, Simon.

Hernández Solís, M.; Fuente Sánchez, D.; Pra Martos, I. (2009). Innovación docente en matemática financiera. [en línea] Disponible en http://www.eduonline.ua.es/ jornadas2010/comunicaciones/185. pdf). [consulta 2012, 13 de abril].

Herrador Alcaide, T. C.; Solís Hernández, M. (2012). Mejora de la calidad docente a través de innovación con redes en la UNED: Aplicación a la docencia en la Contabilidad Financiera. XVII Congreso Internacional para tecnologías para la educación y el conocimiento de tecnologías emergentes. Facultad de Educación. UNED.

Howell, S. L.; Williams, P. B.; Lindsay, N. K. (2003). Thirty-two Trends Affecting Distance education: An Informed Foundation for Strategic Planning. Online Journal of Distance Learning Administration, VI (III), State University of West Georgia, Distance Education Center.

Knowles, M. S.; Holton, E. F.; Swanson, R. A. (2005). The adult learner: The definitive classic in adult education and human 
resource development. San Diego, CA: Elsevier Inc.

Kozma, R. (1986). Implications of instructional psychology for the design of educational television. Educational Communication and Technology Journal, 34 (1), (11-19).

Kregor, G.; Breslin, M.; Fountain, W. (2012). "Experience and beliefs of technology users at an Australian university: Keys to maximizing e-learning potential”. Australasian Journal of Educational Technology, 28 (8), (1382-1404).

LeNoue, M.; Hall, T.; Eighmy, M. A. (2011). Adult Education and the Social Media Revolution. Adult Learning, 22 (2).

Levy, Y. (2007). Comparing dropouts and persistence in e-learning courses. Computers \& Education, 48, (185-204).

Lewis, W.; Agarwal, R.; Sambamurthy, V. (2003). Sources of influence of beliefs about information technology use: An empirical study of knowledge workers. MIS Quarterly, 27 (4), (657-678).

Liaw, S-S. (2008). Investigating students' perceived satisfaction, behavioural intention, and effectiveness of learnings: A case study of blackboard system. Computers and Eduction, 51, (864-873).

López Pérez, M. V.; Pérez López, M. C.; Rodríguez Ariza, L. (2013). Aplicación del aprendizaje combinado en contabilidad. Un análisis comparativo entre diferentes titulaciones universitarias. Revista de Educación, 360.

MacKeracher, D. (2004). Making sense of adult learning ( $2^{\text {nd }}$ ed.). Toronto, Canada: University of Toronto Press.

Marriott, N.; Marriot, P.; Selwyn, N. (2004). Accounting undergraduates' changing use of ICT and their views on using the Internet in higher education - a research note. Accounting Education: An International Journal, 13 (1), (17-130).

Mezirow, J. (1991). Transformative dimensions of adult learning. San Francisco, CA: Jossey-Bass.
Michaledou, A.; Economides, A. (2003). Elearning: Towards a collaborative educational virtual environment. Journal of Information Technology Education, 2, (131-152).

Ong, C.S.; Lai, J.Y.; Wang, Y. S. (2004). Factors affecting engineers' acceptance of asynchronous e-learning systems in high-tech companies. Information \& Management, 6 (6), (795-804).

O’Neill, K.; Singh, G.; O’Donoghue, J. (2004). Implementing e-learning programmes for higher education: A review of the literature. Journal of Information Technology Education, 3, (313-323).

Peltier, J. W.; Drago, W.; Schibrowsky, J. A. (2003). Virtual communities and the assessment of online marketing education. Journal of Marketing Education, 25 (3), (260-276).

Piccoli, G.; Ahmad, R.; Ives, B. (2001). 2Webbased virtual learning environments: A research framework and a preliminary assessment of effectiveness in basic IT skills training. MIS Quarterly, 25 (4), (401-426).

Pituch, K.; Lee, Y.K. (2006). The influence of system characteristics on e-learning use. Computers \& Education, 47 (2), (222244).

Reynard, R. (2007). Hybrid learning: Challenges for teachers. The Journal. Retrieved December 9.

Robert A. E.; Ginns, P.; Piggott, L. (2009). E-learning in higher education: some key aspects and their relationship to approaches to study. Higher Education Research \& Development, 28 (3), (303318).

Roca, J. C.; Gagne, M. (2008). Understanding e-learning continuance intention in the workplace: A self-determination theory perspective. Computers in $\mathrm{Hu}$ man Behavior, 24, (4), (1585-1604).

Roca, J. C.; Chiu, C. M.; Martínez, F. J. (2006). Understanding e-learning continuance intention: An extension of the 
Technology Acceptance Model. International Journal of Human Computers Studies, 6 (8), (683-696).

Santamaría Lancho, M.; Sánchez-Elvira Paniagua, A. (2009). La organización de la docencia en la UNED mediante la colaboración entre equipos docentes y tutores con el apoyo de las tecnologías, en respuesta a las demandas metodológicas del EEES. Seminario de reflexión RED$U$-USC: La coordinación mediante equipos docentes en ES: fortalezas, recursos y necesidades. Santiago de Compostela. [en línea] Disponible en http://congresos.um.es/redu/compostela2009/ paper/viewFile/2971/2901. [consulta 2012, 23 de abril].

Selim, H. M. (2007). Critical success factors for e-learning acceptance: Confirmatory factor models. Computers \& Education, 49, (396-413).

Sharpe, R.; Benfield, G. (2005). The student experience of e-learning in higher education: A review of the literature. Brooks e Journal of Learning and Teaching, 3, (1-10).

Sharpe, R.; Benfield, G.; Roberts, G.; Francis, $\mathrm{R}$. (2006). The undergraduate experience of blended e-learning: A review of $U K$ literature and practice.

Smith, S.; Caruso, J. (2010). The ECAR study of undergraduate students and information technology, EDUCAUSE Center for Applied Research.

Sorensen, C.; Baylen, D. M. (1999). Interaction in interactive television instruction: perception versus reality. Proceedings of the Annual Meeting of the Ameri- can Educational Research Association, Montreal, Quebec, Canada.

Sun, J. N.; Hsu, Y. C. (2012). An experimental study of learners perceptions of the interactivity of web-based instruccion. Interactive with Computers, 24, (35-48).

Sun, P. C.; Chen, H. K.; Lin, T. C.; Wang, F. S. (2008). A design to promote group learning in e-learning: Experiencies from the field. Computers and Education, 50, (661-677).

Wagner, E. D. (1994). In support of a functional definition of interaction. The American Journal of Open distance learning, 8 (2), (6-29).

Wagner, E. D. (1997). Interactivity: From agents to outcomes. In Cyrs, T. E. (Ed.). Teaching andlearning at a distance: What it takes to effectively design, deliver, and evaluate programs. San Francisco: Jossey-Bass Publishers.

Wang, W.; (2009). Investigating the determinats and age and gender differences in the acceptance of mobile learning. British Journal of Educational Technology, 40 (1), (92-118).

Weston, T. J.; Barker, L. (2001). Designing, implementing, and evaluating web-based learning modules for university students. Educational Technology, 41 (4), (15-22).

Zhang, D. A.; Zhou, L. A.; Robert, O.; Briggs, B.; Nunamaker, J. F. (2006). Instructional vídeo in e-learning: Assessing the impact of interactive vídeo on learning effectiveness. Information \& Management, 43, (15-27).

\section{PERFIL ACADÉMICO Y PROFESIONAL DE LAS AUTORAS}

Teresa Carmen Herrador Alcaide. Doctora en Ciencias Empresariales. Profesora Titular de Universidad en la Universidad Nacional de Educación a Distancia (UNED). Premio extraordinario de doctorado. Investigación en contabilidad y gestión del conocimiento en entornos virtuales. Mención honorífica en los IV Premios del Consejo Económico y Social de la CAM. Directora de Tesis, Tesinas y 
Trabajos Fin de Máster. Publicaciones en revistas. Autora de libros. Directora de una Red de Innovación Docente en la investigación desarrollada por la UNED.

E-mail: therrador@cee.uned.es

Montserrat Hernández Solís. Doctora en ciencias Empresariales. Profesora en la UNED. Beca predoctoral de investigación en la Fundación Caja-Madrid. Publicaciones en economía financiera, actuariales y gestión virtual del conocimiento. Codirectora de una Red de innovación docente en el marco UNED. Profesora de Master Cuestiones Actuariales (UNED). Profesora en el Máster en Banca y Finanzas de Instituto Tecnológico de Caixa Galicia.

E-mail: montserrath@cee.uned.es

DIRECCIÓN DE LAS AUTORAS

Facultad de CC. Económicas y Empresariales

Universidad Nacional de Educación a Distancia, UNED

Paseo Senda del Rey 11

28040, Madrid, España

Fechas de recepción del artículo: 17/09/12

Fecha de aceptación del artículo: 15/12/12

\section{Como citar este artículo:}

Herrador Alcaide, T. C.; Hernández Solís, M. (2013). Un estudio del e-learning para adultos en educación universitaria a distancia: un análisis estadístico sobre el rendimiento de estudiantes de contabilidad financiera en la UNED. RIED. Revista Iberoamericana de Educación a Distancia, volumen 16, nº 2, pp. 33-57. 